\title{
Quasar Parallax: a Method for Determining Direct Geometrical Distances to Quasars
}

\author{
Martin Elvis and Margarita Karovska \\ Harvard-Smithsonian Center for Astrophysics, Cambridge MA 02138, USA \\ revised: 5 pm, 3 November 2002
}

\begin{abstract}
We describe a novel method to determine direct geometrical distances to quasars that can measure the cosmological constant, $\Lambda$, with minimal assumptions. This method is equivalent to geometric parallax, with the 'standard length' being the size of the quasar broad emission line region (BELR) as determined from the light travel time measurements of reverberation mapping. The effect of non-zero $\Lambda$ on angular diameter is large, $40 \%$ at $z=2$, so mapping angular diameter distances vs. redshift will give $\Lambda$ with (relative) ease. In principle these measurements could be made in the UV, optical, near infrared or even X-ray bands. Interferometers with a resolution of 0.01 mas are needed to measure the size of the BELR in $\mathrm{z}=2$ quasars, which appear plausible given reasonable short term extrapolations of current technology.
\end{abstract}

We propose a method to determine direct geometrical distances to radio-quiet quasars that can measure the cosmological constant, $\Lambda$, with minimal assumptions. The largest scale use of astronomical distances is the measurement of the geometry of the universe. Distance measurements using the brightness of supernovae of type 1a (SN1a) up to a redshift, z 1.5 as 'standard candles' suggest that the universe has a non-zero cosmological constant, $\Lambda$, so that the expansion of the universe is accelerating (Perlmutter et al. 1999, Riess et al. 1998, 2001). Most of the energy density of the universe, $\Omega$, would then lie in this repulsive 'dark energy', $\Omega_{\Lambda} \sim 0.7$, rather than in 'normal dark matter', $\Omega_{m} \sim 0.3$. This measurement may be key to new physics and new cosmology (Carroll 2001). However, like most methods for finding astronomical distances, the SN1a method has inherent uncertainties due to model dependent assumptions, the small size of the effect $(\sim 10 \%)$, and difficulties of measurement (Rowan-Robinson 2002; Wright 2002; Branch et al. 2001; Richardson et al. 2002; Linder 2001; Goobar et al. 2002). The need for a second, independent, means of determining $\Lambda$ is clear and urgent.

The most direct and model independent method for finding astronomical distances involves simple geometry. This method, parallax (Bessel \& Rath 1839), uses the motion of 
the Earth around the Sun to measure the angular displacement of a nearby star, against an effectively unmoving distant background of stars. By this means we use a known length, in this case the Earth's orbital radius (1 AU), and a known angle to solve the isosceles triangle created by these two quantities and so solve for the distance to the star (figure 1a). Here we propose an equivalent geometric method to determine distances to quasars (figure 1b). In this case the triangle is inverted, with the known length being at the distant quasar, instead of at the Earth. The 'standard length' we propose to employ is the size of the quasar broad emission line region (BELR, Peterson 1997), which is known from light travel time measurements. The angle in the triangle $(\theta$, figure $1 \mathrm{~b})$ is that subtended by the same BELR, as measured with an interferometer. By measuring both quantities to a series of quasars at different redshifts the 'angular diameter distance', $D_{A}$, can be mapped out against redshift, $z$. The resulting angular diameter - redshift relation is a basic characteristic of the space-time metric of the universe. From this relation we can determine

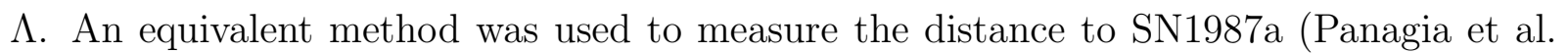
1991, Binney and Merrifield 1998) to 6\%. Because this method uses a 'standard length' rather than a 'standard candle' it is less dependent on physical models and of changes in the fundamental constants, other than $c$, the speed of light. ( $c$ is needed to measure the 'standard length' at the quasar.)

Quasars are highly luminous objects and are readily studied in detail up to the highest redshifts (currently $>6$, Fan et al. 2001). There have been previous proposals to use quasars as distance indicators (Baldwin 1977, Rudge \& Raine 1999, Collier et al. 1999, Homan \& Wardle 2000), but these methods are, to a greater or lesser degree, model dependent. A defining characteristic of quasars is a series of strong broad emission lines from permitted transitions, whose breadths indicate Doppler velocities of 1-5\%c (3000-15,000 km s$\left.{ }^{-1}\right)$. These 'broad emission lines' (BELs) arise from gas that is photoionized by a highly luminous source of radiation with a spectrum that is broader than any single black body, so producing a wide range of ionization in the BELR. Even in radio-quiet quasars, where the observed continuum is not dominated by relativistically beamed jet emission, this continuum is highly variable on short time scales $(\sim 1$ day), and so the source of the continuum is small, $\sim 100 \mathrm{AU}\left(1.5 \times 10^{15} \mathrm{~cm}\right)$ across (Peterson 1997). The response of the BELs to continuum changes is delayed by the time light takes to travel from the continuum source to the BEL emission region. This light travel time gives the size of the BELR (Blandford \& McKee 1982, Peterson 1993, 2001, Netzer \& Peterson 1997). This technique is called 'reverberation mapping'. A typical BELR emitting region radius in nearby active galaxies (for CIV $\lambda 1550 \AA$, Netzer \& Peterson 1997) is 10 light-days $\left(2.5 \times 10^{16} \mathrm{~cm}\right.$, Wandel, Peterson \& Malkan 1999, Kaspi et al. 2000).

In the upper panel of figure 2 we show the angular diameter vs. redshift, $D_{A}$ vs. $z$, 
(a)

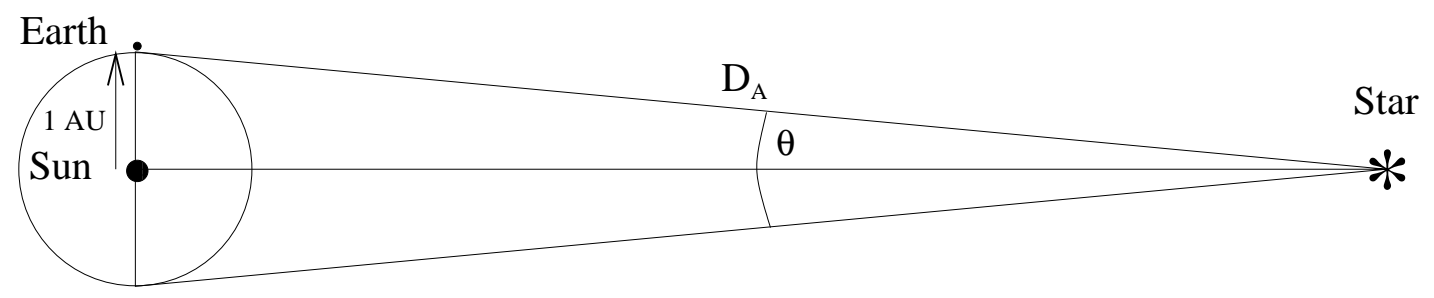

(b)

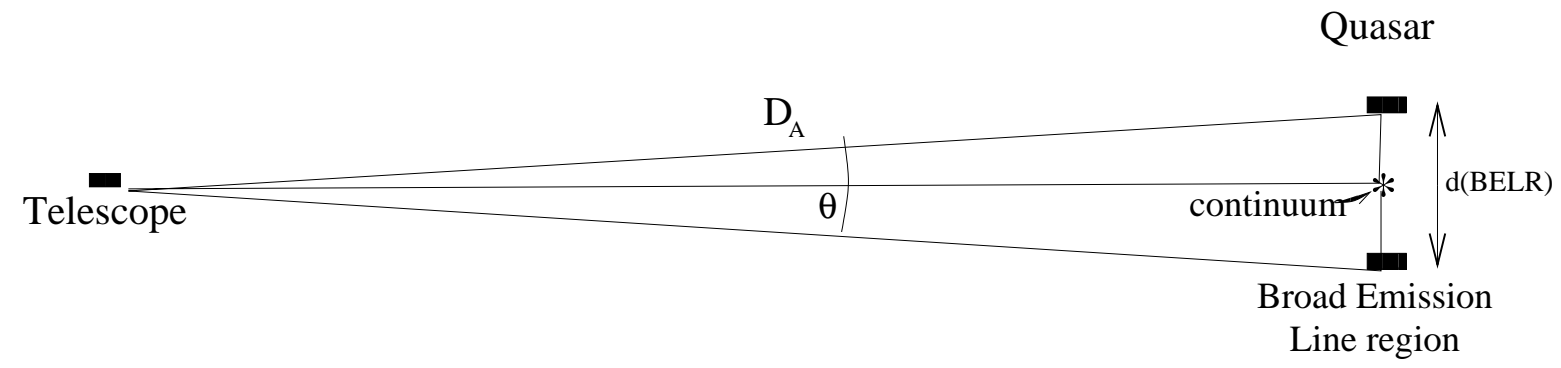

Fig. 1.- Measuring geometric distances to astronomical objects: (a) parallax to a star; (b) broad emission line region distance to a quasar. 
relation for a $\Lambda=0, \Omega=1$ cosmology and in a $\Lambda=0.7, \Omega_{m}=0.3$ cosmology, for a nominal size of 10 light-days. The turn-around in angular diameter at $\mathrm{z} \sim 1-2$ is present in both cosmologies, (and is a feature of all $\Omega=1$ space-times) but is notably weaker in the positive $\Lambda$ model. The crucial value for determining $\Lambda$ is the ratio of $D_{A}$ in the two cosmologies. This ratio is shown in the lower panel of figure 2. The deviation between the two $D_{A}(z)$ relations is a relatively large effect, reaching $40 \%$ by $\mathrm{z}=2$, and then continuing to grow slowly to $55 \%$ at $\mathrm{z}=6$.

To resolve a radius of 10 lt-days at $z>2$ requires 0.001 mas resolution (figure 2 ). This appears to imply a long baseline, $d$, of $100 \mathrm{~km}$ at a wavelength, $\lambda=5000 \AA$, using the Rayleigh criterion $(\theta=1.22 \lambda / d)$. This is currently a demanding requirement, though by no means an unachievable one (Labeyrie 1999). Fortunately quasar BELRs at $\mathrm{z}=2$ are significantly larger than those nearby. This is because the radius of the BELR, $r_{B E L R}$, increases with quasar luminosities, $L$, as $L^{0.5}$ (Wandel, Peterson \& Malkan 1999), and $L$ evolves with redshift as $(1+z)^{3}$ (Boyle et al. 1988, 2000), so $r_{B E L R} \propto(1+z)^{1.5}$. Hence at $\mathrm{z}=3$ a typical quasar (i.e. one at the break luminosity, $L^{*}$, in the optical luminosity function) has a BELR 8 times larger than one at $\mathrm{z}=0$, with typical diameters at $z=2-3$ of $\sim 0.01$ mas. Although this is still a demanding resolution, quasar evolution gives an order of magnitude reduction in the baseline of the interferometers required, from $5 \mathrm{~km}$ to $1 \mathrm{~km}$ at $5000 \AA$.

At low redshifts $(z \sim 0.2)$ the BELR angular diameters are some ten times larger (0.05-0.15 mas, figure 3). Nearby active galaxies would not give a measurement of $\Lambda$, but would determine $H_{0}$, independent of all other 'distance ladder' methods (e.g. Freedman et al. 2001). Diameters of $\sim 0.1$ mas can be measured using a $\sim 200$ meter baseline in the UV (at CIV $1549 \AA \times 1.2$ ), or $\sim 500$ meters in the optical (at $\mathrm{H} \beta 4861 \AA \times 1.2$ ).

The choice of strong BELs to measure is reasonably limited (Osterbrock 1989): Ly $\alpha$

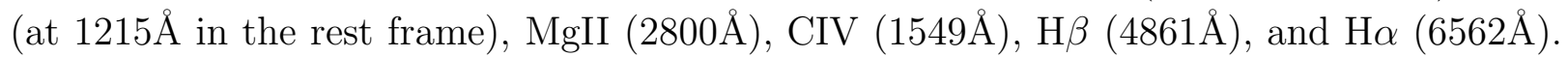
The hydrogen lines and CIV vary quickly, while MgII varies more slowly, in response to

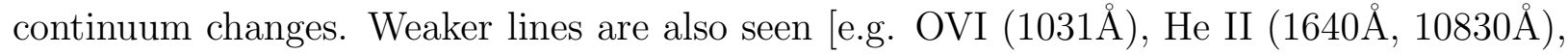
$\mathrm{H} \mathrm{Pa}-\alpha(18751 \AA), \mathrm{H} \mathrm{Pa}-\beta(12818 \AA)]$ and would be candidates under special constraints of redshift and observing band. If the strong narrow X-ray Fe-K emission line in active galaxy spectra at $6.4 \mathrm{keV}$ comes from a BELR sized region or larger, as seems increasingly likely (Chiang et al. 2000, Elvis 2000, Takahashi et al. 2001), then X-rays too would become attractive for measuring $\Lambda$.

Despite this limited choice of emission lines, the optimum wavelength band of the interferometer to use is not obvious. The observed wavelength of any line increases as $(1+z)$, so that the baseline of an interferometer that can resolve the BELR increases with 


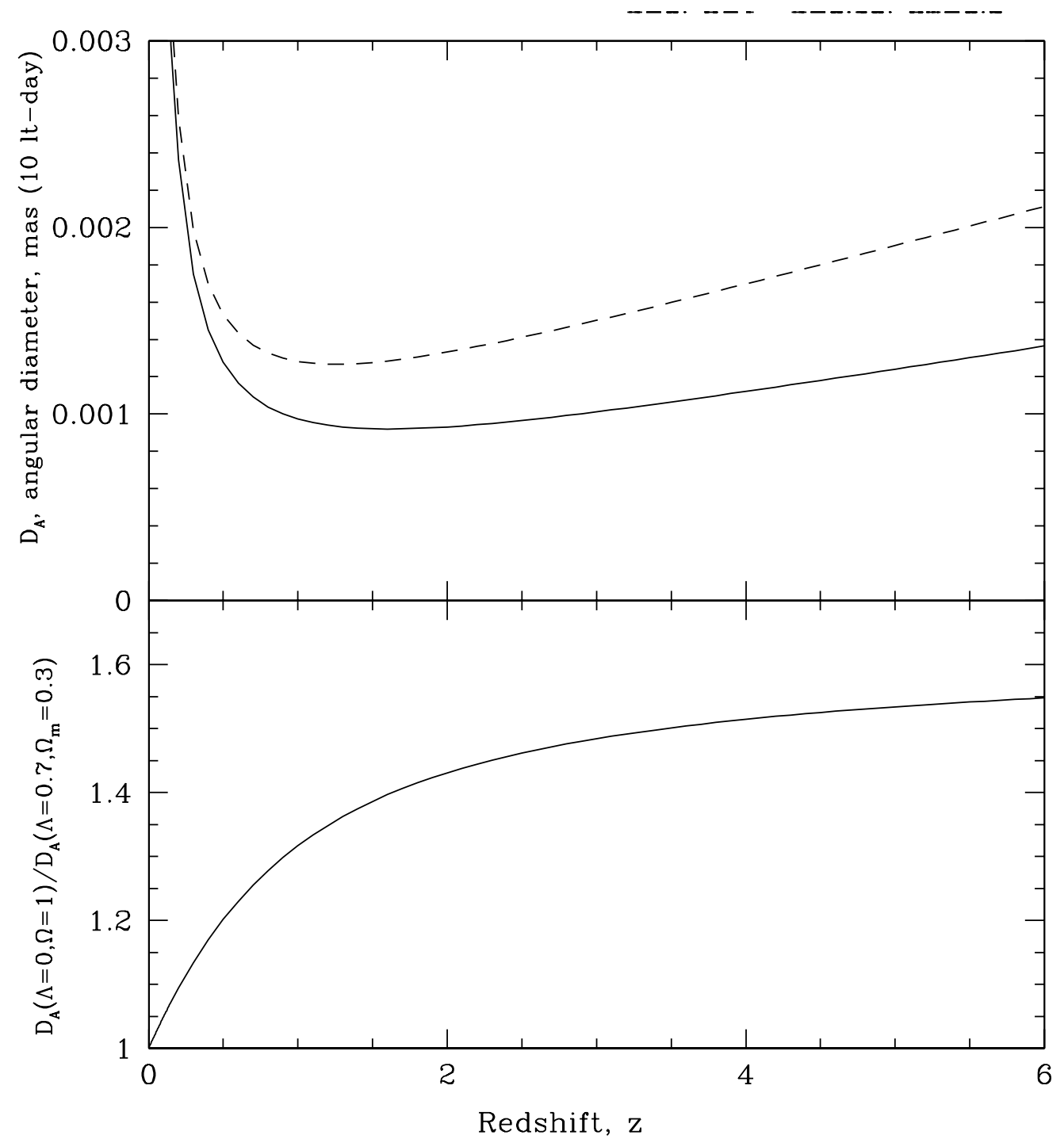

Fig. 2.- top: angular diameter of a 10 light-day long 'standard length' in milli-arcseconds for two cosmologies: $\Omega=1, \Lambda=0$ (dotted line), $\Lambda=0.7, \Omega_{m}=0.3$ (dot-dash line). bottom: ratio of angular diameter in an $\Omega=1, \Lambda=0$ cosmology to that in a $\Lambda=0.7, \Omega_{m}=0.3$ cosmology. 


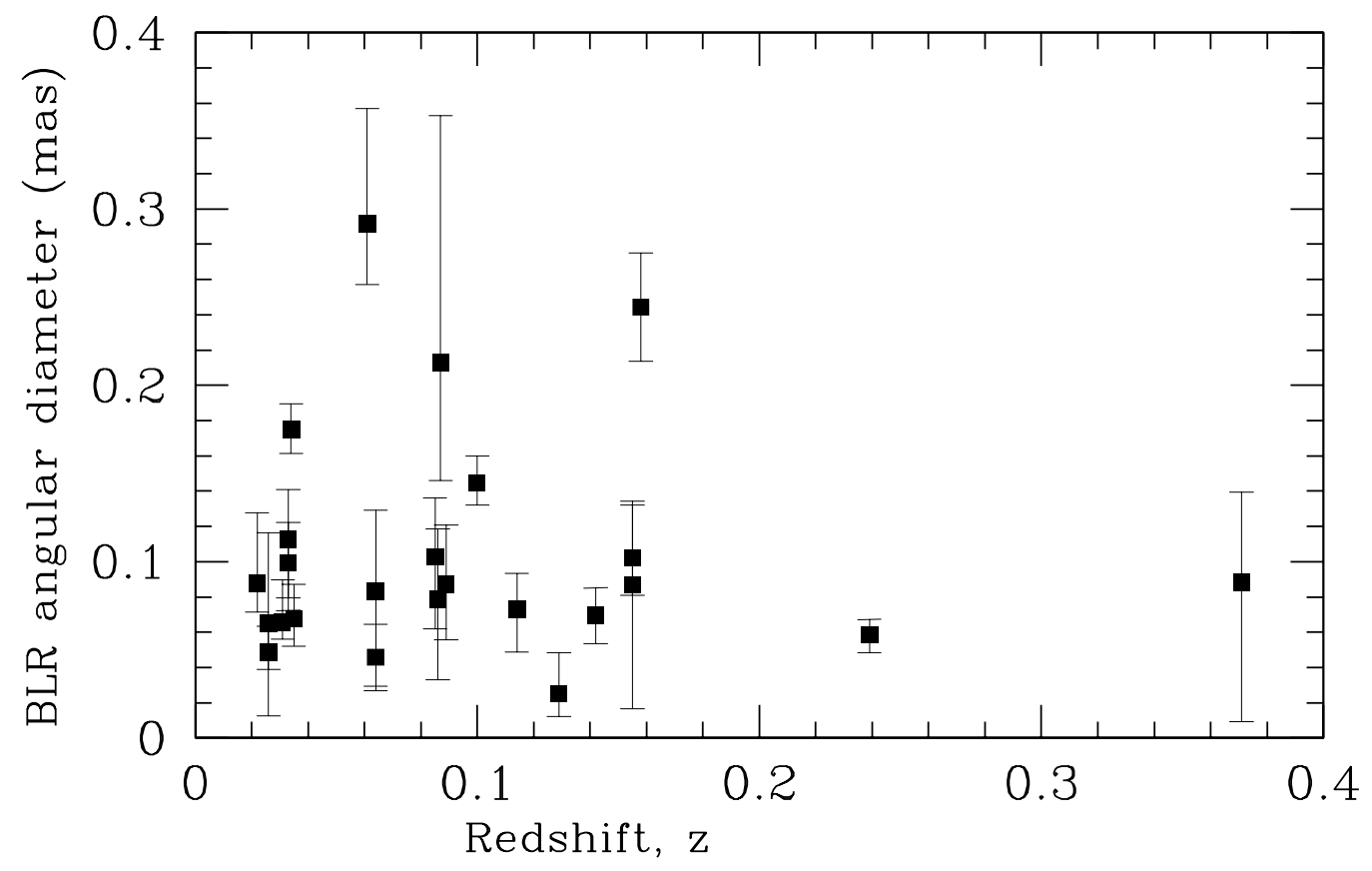

Fig. 3.- Angular diameters for the $\mathrm{H} \alpha$ and $\mathrm{H} \beta$ BELRs of nearby active galaxies, assuming $\mathrm{H}_{0}=65 \mathrm{~km} \mathrm{~s}^{-1} \mathrm{Mpc}^{-1}$ (Peterson et al. 1998, Kaspi et al. 2000). (The values of $\Omega$ and $\Lambda$ are unimportant at these low redshifts.) 
$z$ for a fixed physical size. So in general shorter wavelength emission lines are preferred. However, when these lie short-ward of $3000 \AA$, in the UV or X-ray bands, they can be observed only from space. Several long-baseline space-based interferometers are currently in the planning stages. These include interferometry projects under development such as the Stellar Imager (Carpenter, K. et al 2002), with a 0.1 mas resolution at UV wavelengths , and long term concepts such at the Terrestrial Planet Imager with $6000 \mathrm{~km}$ baselines. 1999) In the X-ray domain baselines $\sim 1000$ times shorter than in the optical can be used: $\sim 10 \mathrm{~m}$ for $\sim 0.01$ mas resolution at $2 \mathrm{keV}$ (i.e. the energy of the Fe-K line at $z=2-3$ ). A diffraction limited version of the Chandra X-ray observatory could directly resolve $\sim 20$ mas. Building such a telescope may be feasible (L. Vanspeybroeck, 2002 private communication). However, to measure $H_{0}$ and $\Lambda$ would require X-ray interferometry, which is under development (Cash et al. 2000).

From the ground, interferometry is easier at longer wavelengths because the atmosphereric patches that limit angular resolution are larger there. The near infrared (1-2 $\mu \mathrm{m}$, the JHK bands) is especially promising, but requires 10 times longer baselines than in the UV. However, several ground-based optical and near-IR interferometers employing large aperture telescopes (e.g. 8-m) on baselines of several hundred meters are currently operating or being built (VLT-I, CHARA, Ohana). The telescope sizes needed for interferometry may be large, since interferometers require narrow bandwidths and these narrow bands may sample only a fraction of the emission line profile. (e.g. VLT-I, Petrov et al., 2000; Ohana, Perrin et al. 2000). Finding an optimal practical choice of BEL, redshift interval and observing technique is a complex problem.

Using high-signal to noise visibility measurements and a model brightness distribution, the limiting resolution of a a telescope can significantly exceed the Rayleigh criterion. For example Karovska et al. (1989) measured diameters of several stars and of SN1987A with the CTIO 4-m telescope, and obtained angular diameters of 5-10 mas, with uncertainty of \pm 1 mas. I.e. as much as 5 times smaller than the the Rayleigh diffraction limit ( 30 mas at optical wavelengths). Similarly, the HST Fine Guidance Sensors (FGS) have been used to measure the sizes of objects to scales of 8 mas, well below the $\sim 50$ mas optical diffraction limit of HST (e.g. Hook et al. 2000).

Accurate measurements of the reverberation times of fairly high redshift quasars are required to carry out this distance determination project. Since the size of the broad emission line region depends systematically on the line measured, with lower ionization lines coming from larger regions, the lag-time and angular size measurements must be carried out using the same emission line. Moreover different velocities within a line likely come from different locations, so the same velocity slice of the emission lines must be observed by 
both techniques.

Radio-quiet quasars at $\mathrm{z} \sim 2-3$ seem to be sufficiently variable. Optical continuum variations of at least $0.5^{m}$ are seen in at least $40 \%$ of bright $(m<18)$ radio-quiet quasars (Netzer and Sheffer 1982, Maoz et al. 1994), although Kaspi (2002) finds little variability in another sample. Quasars at $\mathrm{z} \sim 2-3$ are also sufficiently bright. Most of the difference between $\Lambda=0$ and $\Lambda=0.7$ cosmologies has been reached by $z=2-3$ and, as these redshifts span the peak of quasar evolution, there are many optically bright ( $B=17-18$ mag) objects to observe at these redshifts (Wisotzki 2000). The time scale of BEL variations increases with redshift as $(1+z)^{2.5}$, since not only does the BELR size increase, but also the intrinsic variation time scale is dilated by $(1+z)$. As a result, even for the fastest varying lines, e.g. CIV, to observe for 3-5 times the typical reverberation time for quasars at $\mathrm{z}=3$ requires observing programs spanning of 3-5 years. While this lengthens the total program, the time span is not prohibitive, and the frequency of observations is less demanding than in the smaller, more rapidly varying, active galaxies so far measured by reverberation mapping (Peterson 2001).

To measure $\Lambda$ well requires a final accuracy of order $10 \%$ (figure 2 ). The error on measured BELR radii is currently quite large, even up to factors of 2 for the moderately large redshift quasars in Kaspi et al. (2000). However this is not intrinsic to the method, and the smallest error derived so far is $8 \%$ (Peterson et al. 1998). The lag time error is a function of observation density, spectral signal-to-noise ratio and calibration accuracy, and of catching a quasar in a strong outburst. For example, the 1993 HST campaign on NGC 5548 found only weak $(75 \%)$ continuum variations, but had the campaign lasted twice as long ( $\sim 80$ days) a factor 2.5 change would have been found, leading to much improved reverberation lag measurements. A factor 4.5 variation was recorded in an earlier, less intensive, campaign (Clavel et al. 1991) on the same object. Dedicated observing facilities may be required to measure $\Lambda$ by this method.

To achieve $5 \%$ accuracy in lag times, and so leave room for an $8 \%$ error in measuring $\theta$, requires sampling at 0.02-0.1 the BELR light crossing time, $r_{B E L R} / c$ (Peterson et al. 2002, Edelson et al. 2001). Several lag times must be sampled to give an unambiguous result (Horne et al. 2002), giving a nominal minimum of order 200 observations per quasar, i.e. of order once per week at $z=2-3$. These estimates are probably somewhat optimistic, and a well designed program would exceed them by a factor of a few. Large telescopes are not needed to measure high redshift quasar reverberation times. At $z=2$ the 9.9 times longer lag times helps to compensate for the 100 times decreased flux compared with $z=0.2$. A 1.5 meter telescope is enough to achieve a signal-to-noise of 100 in the CIV line at this sampling rate, even allowing for the slight weakening of the high ionization emission lines 
with luminosity (Baldwin 1977). (This estimate applies to space-based instruments where sources of noise other than counting statistics can be rendered unimportant). Velocity resolved spectra, to match interferometric bands, will require larger telescopes.

Fortunately observations indicate that a single time scale does dominate for each emission line (Netzer \& Peterson 1997). Even so, there is an inherent problem in relating reverberation lag times as currently measured to the angular sizes that an interferometer would measure. Even perfectly sampled data will produce a broadened cross-correlation function $(\mathrm{CCF})$, and it is not obvious which delay time, e.g. the CCF peak, or the CCF centroid, corresponds to the angular size from an image. Moreover, reverberation mapping measures a 'responsivity weighted' size of the light delay surface, while interferometry measures an 'emission weighted' size. That these are different is shown by the fact that the amplitude of variation of the emission lines is much less than that of the continuum. Quite often, factor 2 changes in the UV continuum produce only $20 \%$ changes in the emission lines (e.g. Peterson 2001, figure 31). The high ionization and high order lines vary most strongly, but they also tend to be weak (Ulrich et al., 1991). Not only does this weak response require larger telescopes, but also the gas being studied is not the same in the two types of measurement.

We suggest that this difficulty can be removed by observing the target quasar with an interferometer in both low and high continuum states, with appropriate lags included, so that the difference map will be a measure only of the 'responsive fraction' of the line, i.e. that part which responds to continuum changes. The two methods would then be measuring the same thing. This approach would require monitoring a number of quasars for continuum changes, and then using them to trigger 'target of opportunity' interferometer measurements.

A more ambitious approach would be to monitor quasars with a true imaging interferometer. We could then watch continuum changes propagating outward and creating emission line responses as a function of wavelength. Since the geometry of quasar BELRs is not known this 'imaging reverberation mapping' method would go a long way to removing the ambiguities in the method. Possibilities for the BELR geometry range from a simple orderly wind (Elvis 2000) to a maximally chaotic distribution of clouds (Baldwin et al. 1995). The BELR has 6 dimensions of structure: 3 of position plus 3 of velocity. Imaging of the BELR reverberations in several velocity bands, while the continuum varies would provide 4 observed dimensions (RA, dec, velocity and lag time) directly. If there is an axis of symmetry, then the BELR will be under-determined only by 1 dimension. The angular diameter distances derived would then have minimal room for uncertainty. Currently reverberation mapping measures only $1+$ dimensions (lag time, and minimal velocity 
information), so 4 dimensions would be a great advance.

However, even this method remains 1 dimension short of being fully determined. Long term (years) variations of the BELR may provide the missing 6th dimension. BEL profile changes occur in some objects (e.g. Akn 120, Kollatschny et al. 1981). Moreover, the size of the BELR for particular emission lines seems to respond to changes in the central continuum flux. Lag times in NGC 5548 seem to be longer when the flux is high than when the flux is low (Peterson et al. 1999, Peterson et al. 2002). As a result, the size and lag measurements are best made at the same time. Again, the best program would be the continuous interferometric monitoring mentioned above. A fully solved 6-D BELR structure and kinematics would not only be a major advance in its own right, but would also minimize the uncertainties in the cosmological parameters derived from this method.

Although interferometry is presently not ready to make a measurement of $\Lambda$ using quasar parallax, or perform true imaging of quasar BELRs, ground-based interferometry has reached a stage in which initial determinations of diameters of the BELRs of nearby AGNs is feasible. If the geometry of a sample of nearby AGNs can be explored with these long-baseline interferometers then they will lay the foundations for a long term program to determine cosmological parameters by this method. In the near future sub-milliarsecond resolution will be achieved with the long baseline $(200-800 \mathrm{~m})$ interferometers VLT-I and Ohana in the near-IR, where the Paschen lines are available at low z. In the following few years, imaging should become possible in the optical as well (e.g. at H $\alpha$ ). Imaging of nearby AGN BELRs in the lines used in reverberation measurements will determine their spectral/spatial morphology, and allow the technique to be refined. This will provide a crucial base for 'parallax' measurements of high redshift quasars to determine $\Lambda$, when the next generation of long-baseline interferometers with $<0.01$ mas resolution become available.

We thank Brad Peterson and Rick Edelson for valuable comments on reverberation mapping measurements, Jill Bechtold for the use of her $\Lambda$ cosmology code, Jonathan McDowell for assistance with general relativity, and Helene Sol for informing us of the Ohana project. We thank the anonymous referee for comments on line responsivity which led to a significant improvement in the paper. This work was supported in part by NASA contract NAS8-39073 (Chandra X-ray Center). 


\section{REFERENCES}

Baldwin J.A., 1977, ApJ, 214, 679

Baldwin J.A., Ferland G., Korista K. \& Verner D., 1995, ApJL, 455, L119

Bessel F.W. \& Rath H.G., 1839, Aston. Nach. 16, no.356

Blandford R.D. \& McKee C.F., 1982, ApJ 255, 419

J. Binney and M. Merrifield 1998, Galactic Astronomy

Boyle B.J., Shanks T. \& Peterson B., 1988, MNRAS, 235, 935.

Boyle B.J., Shanks T., Croom S.M., Smith R.J., Miller L., Loaring N. \& Heymans C., 2000, MNRAS, 317, 1014.

Branch D., Perlmutter S., Branch E. \& Nugent P., 2001, astro-ph/0109070

Carroll S.M., 2001, astro-ph/0107571

Carpenter, K. et al 2002, proceedings of SPIE meeting, Hawaii, in press

Cash W., Shipley A., Osterman S. \& Joy M., 2000, Nature, 411, 644.

Chiang J. et al., 2000, ApJ, 528, 292

Clavel J. et al. 1991, ApJ, 366, 64

Collier S., Horne K., Wander I., Peterson B.M., 1999, MNRAS 302, L24

Edelson R., Griffiths G., Markowitz A., Sembay S., Turner M.J.L. \& Warwick R., 2001, ApJ, 554, 274

Elvis M., 2000, ApJ, 545, 63

Fan X., et al., 2001, AJ, 122, 2833

Freedman W., et al., 2001, ApJ, 553, 47

Goobar A., Bergström \& Mörtsell, 2002, A\&A, astro-ph/0201012

Homan D.C. \& Wardle J.F.C., 2000 ApJ 539, 847

Hook, R. N., Schreier, E. J., Miley, G. 2000, ApJ, 536, 208

Horne K., Peterson B.A., Collier S.J. \& Netzer H., 2002, PASP, in press astro-ph/0201182 
Karovska M., Koechlin L., Nisenson P., Papaliolios C., Standley C., 1989, ApJ 340, 435

Kaspi S., Smith P.S., Netzer H., Maoz D., Jannuzi B.T., \& Giveon U., 2000, ApJ, 533, 631.

Kollatschny W., Fricke K.J., Schleicher H., \& York H.W., 1981, A\&A 102, L23

Linder E.V., 2001, astro-ph/0108280

Maoz D., Smith P.S., Jannuzi B.T., Kaspi S. \& Netzer H., 1994, ApJ, 421, 34

Netzer H. \& Sheffer Y., 1983, MNRAS, 203, 935

Netzer H. \& Peterson B.M., 1997, "Astronomical Time Series", eds. D. Maoz, A. Sternberg, and E.M. Leibowitz, 1997 (Dordrecht: Kluwer), p. 85.

Osterbrock D.E., 1989, 'Astrophysics of Gaseous Nebulae \& Active Galactic Nuclei', [Univ. Science Books: Mill Valley]

Panagia N., Gilmozzi R., Macchetto F., Adorf H.-M., \& Kirshner R.P., 1001, ApJ, 380, L23

Perlmutter S., et al., 1999, ApJ, 517, 565

Perrin G., Lai O., Léna P. \& Coudé du Foresto V., 2001, SPIE, SF2-A-2002, eds. F. Combes \& D. Barret, p.98

Peterson B.M., 1993, PASP, 105, 247

Peterson B.M., 1997, 'An Introduction to Active Galactic Nuclei' [Cambridge:CUP]

Peterson B.M., Wanders I., Betram R., Hunley J.F.; Pogge R.W., Wagner, R.M., 1998, ApJ, 501, 82

Peterson B.M. et al., 1999, ApJL, 510, 659

Peterson B.M., 2001, in 'The Starburst-AGN Connection 2001', [Singapore: World Scientific] astro-ph/0109495

Peterson B.M. et al., 2002, ApJ, submitted.

Petrov R. et al., 2000, Proc. SPIE Vol. 4006, 68, 'Interferometry in Optical Astronomy' eds: P.J. Lena and A. Quirrenbach. http://buz.obs-nice.fr/amber//),

Rowan-Robinson M. 2002, MNRAS, 332, 352

Richardson D., Branch D., Casebeer D., Millard J., Thomas R.C. \& Baron T.E., 2001, AJ, 123,745 
Riess A.G. et al., 1998, AJ, 116, 1009

Riess A.G., Nugent P.E., Schmidt B.P., Tonry J., Dickinson M., Gilliland R.L., Thompson R.I., Budavári T., Casertano S., Evans A.S., Filippenko A.V., Livio M., Sanders D.B., Shapley A.E., Spinrad H., Steidel C.C., Stern D., Surace J. \& Veilleux S. 2001, astro-ph/0104455

Rudge C.M. \& Raine D.J., 1999, MNRAS 308, 1150

Takashi K., Inoue H. \& Dotani T., 2002, astro-ph/0204300

Ulrich M.-H., Boksenberg A., Bromage G., Clavel J., Elvius A., Penston M.V., Perola G.C. \& Snijders M.A.J., 1991, MNRAS, 382, 483

Wandel A., Peterson B.M. \& Malkan M.A., 1999, 526, 579

Wisotzki L., 2000, A\&A, 353, 853

Wright E.L., 2002, astro-ph/0201196 